\title{
A technique for fast and safe collection of urine in newborns
}

\author{
Nepal $A^{1}$, Basnet $R^{2}$, Acharya $R^{3}$, Shrestha $S^{4}$, Koirala $S^{5}$, Poudel $A^{6}$, Ojha $A^{7}$, Manandhar $\mathrm{DS}^{8}$
}

${ }^{1}$ Archana Nepal, Lecturer, Patan Academy of Health Sciences; ${ }^{2}$ Rydam Basnet, Resident, Kathmandu Medical College and Teaching Hospital; ${ }^{3}$ Rukma Acharya, Pediatrician, B n C hospital, Jhapa; ${ }^{4}$ Sushma Shrestha, Senior House Officer, Paropakar Maternity and Children's hospital; ${ }^{5}$ Satish Koirala, Resident, ${ }^{6}$ Anshu Poudel, Resident, Kathmandu Medical College and Teaching Hospital; ${ }^{7}$ Anil Ojha, Associate Professor, Kathmandu Medical College and Teaching Hospital; ${ }^{8}$ Dharma Sharna Manandhar, Professor, Kathmandu Medical College and Teaching Hospital, Kathmandu, Nepal.

\begin{abstract}
Background: Urine collection in children especially in new born is a great challenge. A wide range of clinical interventions for urine collection is described in the literature, including non-invasive and invasive methods. Mid-stream urine collection is considered the most appropriate technique for older children. Here we are testing a method for obtaining mid- stream urine sample in newborns.

Objective: To test a technique of urine collection for obtaining mid-stream urine sample in newborns.

Method: A prospective feasibility study of a technique of urine sample collection based on bladder and lumbar stimulation maneuvers done in 100 newborns of less than 28 days of life over a period of one month. The main variable was the success rate in obtaining a midstream urine sample collection within four minutes and secondary variables were time required to obtain the sample and associated complications.

Results: Mid- stream urine sample was collected successfully in $91 \%$ of babies. Mean time required for urine collection was 59.7 seconds with standard deviation of 46.4 seconds and median time was 47 seconds. No untoward complication other than controlled crying was seen.

Conclusion: This is a quick and safe technique for mid-stream urine sample collection in newborn with high success rate and minimal discomfort.
\end{abstract}

Key words: Lumbosacral massage, Neonates, Urine

\section{INTRODUCTION}

T T rine collection in children especially in new born is a great challenge. A wide range of clinical interventions for urine collection is described in the literature, including non-invasive and invasive methods. The most common non-invasive technique is urine collection using sterile bags, which is associated with significant patient discomfort and contamination of samples ${ }^{1}$. Obtaining a clean-catch urine sample is the recommended method for urine collection in children who are able to co-operate. However, in children lacking sphincter control, clean catch urine is difficult and time-consuming. Hence many a time invasive methods (catheterisation and needle aspiration of urine from the bladder) are used for sample collection.

Address for correspondence

Dr. Archana Nepal

Lecturer

Department of Paediatrics,

Patan Academy of Health Sciences (PAHS), Lalitpur, Nepal

E-mail: archuuana@gmail.com
Micturition reflex involves impulses travelling from bladder to the sacral region of the spinal cord and from sacral region of spinal cord back to the bladder. There is no influence from the higher centre of brain in children who are not continent. Stretch receptors in the bladder initiate the action potential for the reflex' ${ }^{1}$. We are using a manoeuvre first described by Herreros Fernandez ${ }^{2}$ that utilizes this theory to collect mid-stream urine sample in neonates and determine the success rate and safety of this non-invasive technique to obtain clean-catch urine samples in new born.

\section{METHODS}

This is a prospective study carried out at Kathmandu medical college teaching hospital, neonatal intensive care unit and postnatal ward. This study was carried out over one month period (January 2014). Study population was newborn less than 28 days of age with sample size of 100. Sick newborns (poor feeding, dehydrated, any medical condition that ruled out manipulation) were excluded from the study. 


\section{TECHNIQUE}

Three people are needed to perform the procedure - one person holds the newborn as show in Figure 1, the next one carries out the procedure and the third person notes the time. Trained doctors performed the procedure. First the child was given breast feeding or formula feed as per her/his weight and day of life. Feeding was done by NICU trained nurses. Twenty- five minutes after feeding, the infant's genitals were cleaned thoroughly with warm water and soap and dried with sterile gauze.

Neonate is then held under her/his armpits with their legs dangling and a sterile collector is placed near the baby in order to collect urine. Bladder stimulation is done by gently tapping in the supra-pubic region at a frequency of hundred taps or blow per minute for a minute.

This is followed by stimulation of the lumbar paravertebral zone in the lower back with a light circular massage for 30 seconds. Both stimulation manoeuvres are repeated (suprapubic tap total three cycles and back massage total two cycles) (Figure2).

Success is defined as the collection of urine sample within four minutes of starting the stimulation manoeuvre (Figure 3). Secondary variables were the time taken to obtain the sample and complications. The sample collection time was defined as the time from the beginning of the stimulation procedure (ie, the tapping) to the beginning of sample collection.

Data compilation and analysis was done using Statistical Package for Social Sciences (SPSS) version 16.0.

\section{RESULTS}

A total of 100 newborns were included in the study, 59 male and 41 female. Mean age of the sample population was 31.5 hours. We had success rate of $91 \%$. Mean time for sample collection was 59.7 seconds with a standard deviation (SD) of 46.4 seconds, median time 47 seconds and mode of 40 seconds. Majority (96.6\%) of males passed urine and $90.2 \%$ females passed urine. Mean time required for passing urine in male is 55.21 seconds with SD of 45.51 seconds and for female is 67.18 seconds with SD of 47.7 seconds. All 91 newborn who passed urine voided during supra-pubic tap. There were not any untoward complications except for crying which would stop with nonnutritive sucking or holding the baby in lap for few seconds.

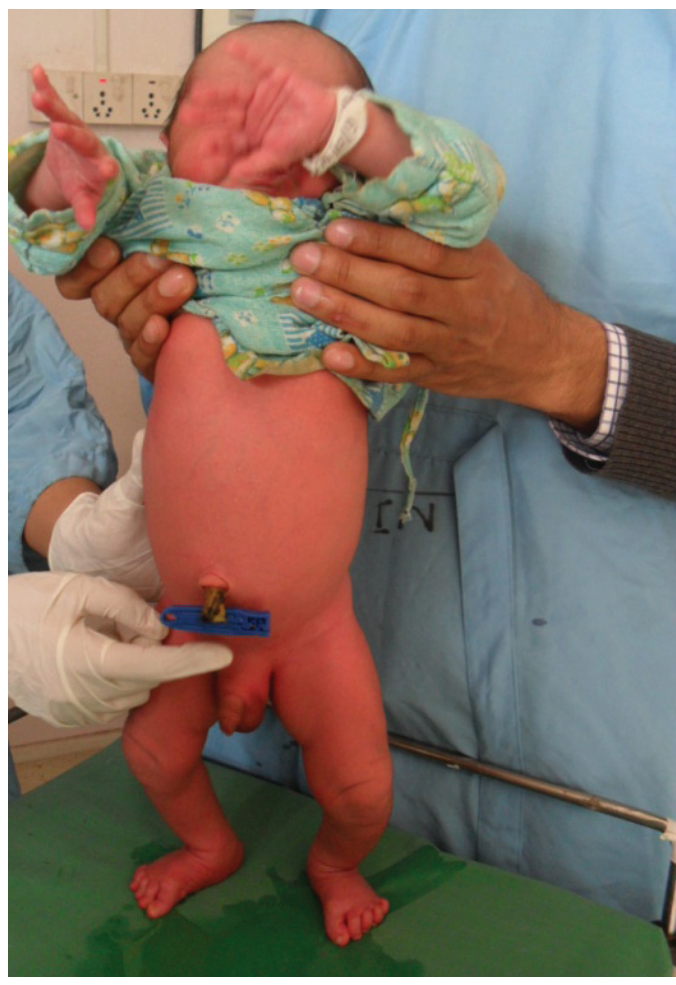

Figure 1: Neonate held under his armpits with his legs dangling and suprapubic tap

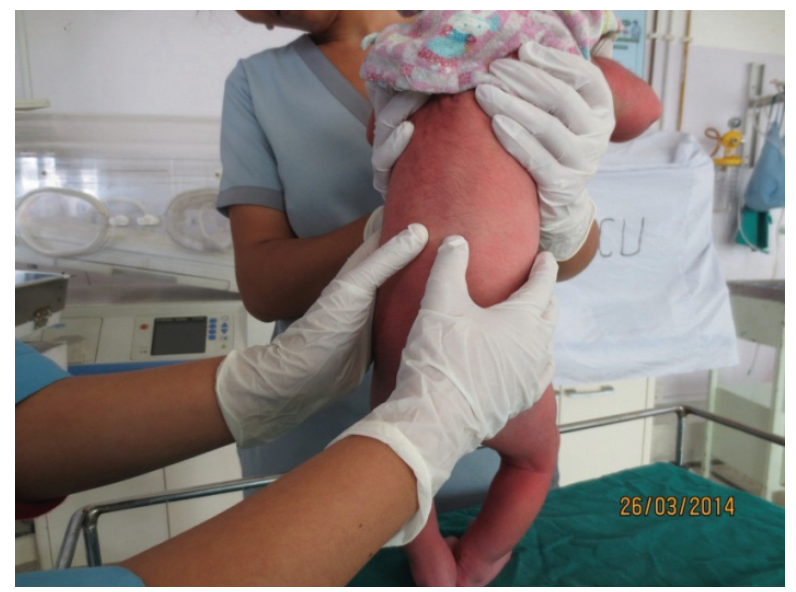

Figure 2: Lumbosacral massage

\section{DISCUSSION}

Collection of urine sample in neonates and infants has always been difficult. Invasive methods of sample collection (suprapubic aspiration, catheterization) are painful and difficult due to irregular voiding pattern. Spontaneous urine sample collection is time consuming and tedious. Hence a method of mid- stream urine sample collection involving suprapubic tap and lumbosacral massage is worthwhile in children who have not attended continence ${ }^{3}$. 


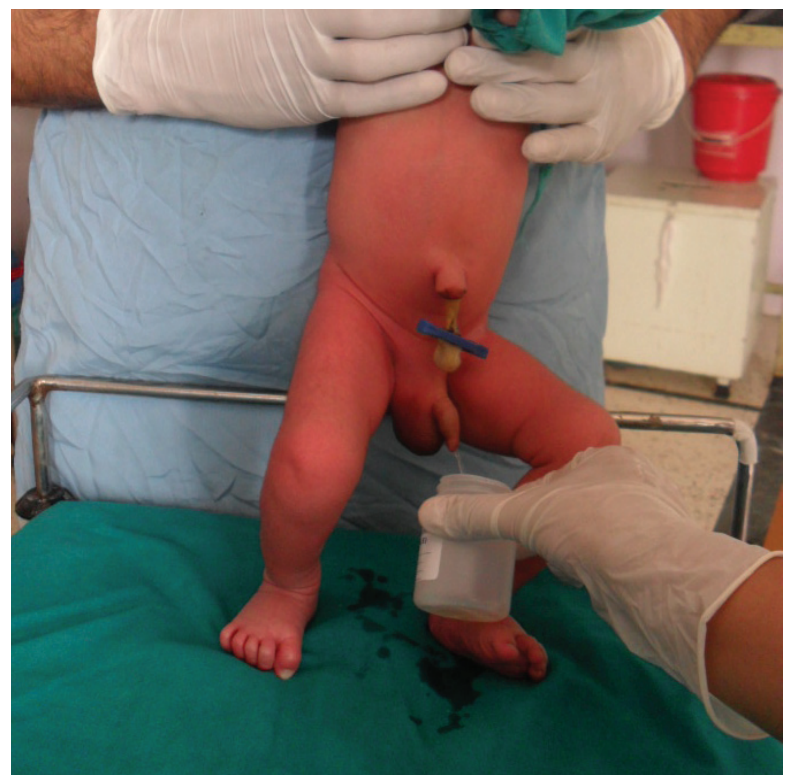

Figure 3: Mid- stream urine being collected

This maneuver exploits the theory that micturition is a simple arch reflex in children not attaining continence. Mechanical stimulation of stretch receptors of bladder in such children can initiate this reflex ${ }^{2}$. This technique has high success rate and a mean time for passing urine of less than $1 \mathrm{~min}$. Herreros Fernandezs showed success rate of $86 \%$ and mean urine collection time of 57 s employing this technique. Our result is similar to theirs with success rate of $91 \%$ with mean time for urine collection 59.7 seconds 4 .
Altuntus $\mathrm{N}$ et al did a case control study utilizing similar technique for experimental group and wait and watch for control group ${ }^{2}$. The success rate of urine collection was significantly higher in the experimental group (78\%) than in the control group (33\%; $\mathrm{p}<0.001)$. The median time for sample collection was 60 seconds in the experimental group and 300 seconds in the control group $(p<0.0001)$. Antoine Tran also utilized bladder stimulation technique for urine sample collection and had success rate of $55.6 \%$, median time of 52.0 seconds $(10.0 ; 110.0)^{5}$. He also found that the success rate decreased with age from $88.9 \%$ (newborn) to $28.6 \%$ ( $>1 \mathrm{y})$ ( $\mathrm{p}=$ $0.0001)$ and with weight, from $85.7 \%(<4 \mathrm{~kg})$ to $28.6 \%$ $(>10 \mathrm{~kg})(\mathrm{p}=0.0004)$.

In our study we saw that all children passed urine during supra-pubic tap raising the question on need of lumbosacral massage in the maneuver. The main limitation of our study was lack of control group and also the sample population included was neonates with mean age of 24 hours making it difficult to generalize the result for late neonates.

\section{CONCLUSION}

This technique allows fast and safe collection of midstream clean-catch urine without any untoward effect on the newborn. Hence this technique can be used as a primary method of urine sample collection in neonatal ward.

\section{REFERENCES}

1. National Institute for Health and Care Excellence. NICE guidelines for UTI in under 16s: diagnosis and management. NICE guidelines CG54:2007.

2. Hall JE, Guyton AC. Guyton and Hall Text book of medical physiology.12th edition. Philadelphia, Pennsylvania: Elsevier Inc; 2010.

3. Herreros Fernández ML, González Merino N, Tagarro García A, de la Serna Martínez M, Contreras Abad MT, et al. A new technique for fast and safe collection of urine in Newborn.Arch Dis Child. 2013; 98(1):27-9.

4. Altuntas N, Tayfur AC, Kocak M, Razi HC, Akkurt S. Midstream clean- catch urine collection in newborn: a randomized controlled trial. Eur J Pediatr. 2015;174(5):577-82.

5. Tran A, Fortier C, Giovannini-Chami L, Demonchy D, Caci H, Desmontils J, et al. Evaluation of bladder stimulation technique to collect midstream urine in infants in a pediatric emergency department. PLoS One. 2016;11(3):e0152598. 